\title{
A case of heparin induced thrombocytopenia managed by Rivaroxaban
}

\author{
Ali Eshraghi ${ }^{1}$ and Faeze Keihanian ${ }^{1}$ \\ ${ }^{1}$ Mashhad University of Medical Sciences
}

November 29, 2021

\begin{abstract}
Heparin-induced thrombocytopenia (HIT) is an immunogenic disorder. It can lead to thrombocytopenia and a hypercoagulated state with an increased risk for new thrombosis. We here reported a 49-year-old man with previous cardiac surgery and heparin administration, treated by new oral anticoagulant agent, Rivaroxaban.
\end{abstract}

\section{Introduction}

Unfractionated heparin (UFH) and the low molecular weight heparins (LMWHs) are the current standard of care for patients requiring short-term anticoagulation (1). Clinicians use heparin for prophylaxis of thrombosis or treatment in various clinical situations (2). Patients of any age, receiving any type of heparin at any dose by any route of administration, are at risk of developing anti PF4 (ABs) antibodies. However, they will all not necessarily develop the clinical syndrome of Heparin Induced Thrombocytopenia (HIT) (3). HIT is a syndrome determined by starting with fall in platelet count (50\% from baseline) in 5 to 10 days of drug administration. Thrombosis can be happened during early days after thrombocytopenia and its prevalence in these patients is nearly $50 \%$ (4). HIT is a severe potentially life-threatening complication (4). There are some alternative anticoagulants for treating HIT: Danaparoid, Lepirudin and Argatroban, those are activated factor $\mathrm{X}$ inhibitors or direct thrombin inhibitors. However, they have many limitations such as higher price, need to hospital stay, monitoring and also making serious bleeding (5). Recent trend in using oral direct factor Xa agents is increasing because of their lack of interaction with cationoic protein platelet factor 4 (PF4) (6). We reported a HIT patient with previous coronary artery bypass graft (CABG) that his thrombosis was treated by Rivaroxaban.

\section{Case presentation}

The patient was a 49-year male with no history of underlying disease admitted due to acute coronary syndrome. He was presented with exertional typical chest pain. We found multi-vessel coronary disease during coronary angiography. Patient underwent CABG and 5 days later, he discharged. Two weeks after discharge, he re-admitted for his resting dyspnea. He was hemodynamically stable. In his ECG there was a new Q wave in inferior leads. Trans thoracic echocardiography (TTE) was performed for him and demonstrated left ventricular ejection fraction (LVEF) of 25\% (prior TTE after CABG showed EF of 55\%). There was new regional wall motion abnormality in infero-posterior of left ventricle (LV) wall. Another important finding in TTE was large fresh thrombus in LV apex. His platelet count was 100,000 that was significantly decreased from prior admission. Unfractionated heparin was started for him by adjusting to his weight along with other medication including Aspirin and Clopidegrol. In the second day after readmission platelet count changed to 90,000 . Platelet was checked in $3^{\text {rd }}$ day and it was $84,000 \mathrm{mg} / \mathrm{dL}$. We asked a hematologic consultation. Peripheral blood smear was performed and showed low platelet count. Heparin induced thrombocytopenia was our leading suspicion because of drop of platelet count, evidence of 
thrombosis, history of surgery and receiving heparin. We did not have any direct thrombin inhibitor in our center. Renal function tests were normal. Liver function tests were mildly elevated due to hepatic congestion (AST: $60 \mathrm{IU} / \mathrm{L}$ and ALT: $40 \mathrm{IU} / \mathrm{L}$ ). So, we decided to begin Rivaroxaban as an anticoagulant. Patient was informed about the rationale of this decision. ASA and Clopidegrol were hold. Rivaroxaban (Axabinß) was administered $15 \mathrm{mg}$ /daily beside checking out liver function test and platelet count daily. Two days after UFH discontinuation, platelet count was elevated to $105,000 \mathrm{mg} / \mathrm{dL}$ and LFT reversed to normal range. The patient discharged one week after use of Rivaroxaban. Platelet count at the time of discharge was $150,000 \mathrm{mg} / \mathrm{dL}$ and $\mathrm{EF}$ was $45 \%$. General condition of patient was good and ASA was re-administer for him. Repeated echocardiography 2 weeks after admission revealed significant reduction in LV clot size. In three months follow up, patient had normal examination and platelet count was $205,000 \mathrm{mg} / \mathrm{dL}$ and there was no LV clot in TTE.

\section{Discussion}

In this patient, history of ACS ,UFH administration and history of CABG and platelet drop after rebegining of UFH along with thrombotic event (Left Ventricular (LV) clot and evidence of saphenous vein graft (SVG) occlusion) strongly suggested HIT. We also contributed the evidence of new Q wave and falling EF to thrombotic event in a SVG that was resulted from HIT. So, we managed him as a case of HIT without specific test for HIT. To our knowledge based on literature there are few case report in using Rivaroxaban for the treatment of HIT and it is the first experience in Iran.

Rivaroxaban is a suitable candidate for treatment of HIT that can be alternate with other non-heparin anticoagulant. Its route of administration orally by fixed dosing with no requirement of coagulation monitoring and proved efficacy in the treatment of venous and arterial thromboembolism in other settings, made it an ideal choice (8). There are some case reports those present the use of Rivaroxaban for treatment of HIT. In our patient, after discontinouing heparin and Rivaroxaban, the platelet count was developed and patient discharged without any side effect. It is because that Rivaroxaban in contrast with heparin and heparin-like agents, directly inhibit activated clotting factors Xa or IIa and does not act with PF4; So HIT will not be induced (1). Other advantages of using Rivaroxaban is its rapid onset in action and no effect on protein $\mathrm{C}$ or S that can prevent hypercoagulability (7). Sartori et al. described a case of HIT treated with rivaroxaban. They presented a 68 year-old man with a thrombosis confined to the internal gastrocnemius and soleal veins. HIT was induced during administration of enoxaparin $80 \mathrm{mg}$ twice a day. They showed development of platelet count to base line after 6 days from enoxaparin withdrawal and followed up the patient for 3 months without any an uneventful course (8). Linkins et al. in a single arm, multicenter prospective cohort study evaluated the efficacy of Rivroxaban $15 \mathrm{mg} /$ bid on twenty-two adults with suspected or confirmed HIT. They continoued the treatment until platelet recovery, then stepped down to rivaroxaban $20 \mathrm{mg}$ daily until day 30. They showed that platelet recovery was achieved in nine out of 10 HIT-positive patients with thrombocytopenia (9). Sharifi et al. in their prospective study investigated the effect of new oral anticoagulants on the outcome of patients with HIT. They evaluated the outcomes of 22 patients who were treated before by these agents and follwed them up for about 19 months. They reported that no patient developed arterial thrombosis and all of them tolerated oral agents. They used Dabigatran at $150 \mathrm{mg}$ twice daily to 6 patients, Rivaroxaban at $20 \mathrm{mg}$ daily to 11 patients and apixaban at $5 \mathrm{mg}$ twice daily to 5 patients (10). As it can in the literature, although using new oral anticoagulant agents like Rivaroxaban or Dabigatran is yet uncommon for HIT treatment, its usage is going to be increased and it can be helpful for HIT. In our case we did not have definitely laboratory finding that confirmed HIT, however we highly suspicious to HIT by the existence of thrombosis and drop in platelet count.

\section{Conclusion}

We here presented a HIT case for the first time in Iran by a thrombosis in LV, that was treated by Rivaroxaban. Our experience can help the hypothesis in which Rivaroxaban considered as an ideal agent for treating HIT. Rivaroxaban may be a safe and effective drug for stable patient with HIT. Further studies in clinical trial use of this agent are warranted. 


\section{Conflict of interest}

There is no conflict of interest.

We declare that none of the authors listed on the manuscript are employed by a government agency that has a primary function other than research and/or education. None of the authors are submitting this manuscript as an official representative or on behalf of the government.

\section{Acknowledgement}

We all thank nurses of Imam Reza Hospital helped us in different stages of patient evaluations.

\section{Key Clinical Message}

Rivaroxaban and other new oral anticoagulant agents had some priority rather than direct thrombin inhibitors, those are included: lower price, oral instead of parenteral, lack of need for monitoring and having fixed dose.

\section{Authors' contributions}

Ali Eshraghi analyzed and interpreted the patient data regarding the cardiovascular disease and managed patient. Faeze Keihanian helped in management of patient, diagnosis and was a major contributor in writing the manuscript. All authors read and approved the final manuscript.

\section{Date availability statement}

The data that support the findings of this study are available from Imam-Reza Hospital, Mashhad University of Medical Sciences, but restrictions apply to the availability of these data, which were used under license for the current study, and so are not publicly available. Data are however available from the authors upon reasonable request and with permission of the corresponding author.

\section{Patient consent}

The authors have confirmed that patients' consent have been signed and collected in accordance with the journal's patient consent policy.

\section{References}

1. Alquwaizani M, Buckley L, Adams C, Fanikos J. Anticoagulants: a review of the pharmacology, dosing, and complications. Current emergency and hospital medicine reports. 2013;1(2):83-97.

2. Dvorak M, Vlasin M, Dvorakova M, Rauser P, Lexmaulova L, Gregor Z, et al. Heparin and its derivatives in the treatment of arterial thrombosis: A review. Veterinarni Medicina. 2010;55(11):523-46.

3. Coutre S, Leung LL, Tirnauer JS. Management of heparin-induced thrombocytopenia.

4. Ahmed I, Majeed A, Powell R. Heparin induced thrombocytopenia: diagnosis and management update. Postgraduate Medical Journal. 2007;83(983):575-82.

5. Warkentin TE. HIT: treatment easier, prevention harder. Blood. 2012;119(5):1099-100.

6. Hantson P, Lambert C, Hermans C. Rivaroxaban for arterial thrombosis related to heparin-induced thrombocytopenia. Blood Coagulation \& Fibrinolysis. 2015;26(2):205-6.

7. Lee CJ, Ansell JE. Direct thrombin inhibitors. British Journal of Clinical Pharmacology. 2011;72(4):581-92.

8. Sartori M, Favaretto E, Cini M, Legnani C, Cosmi B. Rivaroxaban in the Treatment of Heparin-Induced Thrombocytopenia. Journal of thrombosis and thrombolysis. 2015;40(3):392-4.

9. Linkins L, Warkentin T, Pai M, Shivakumar S, Manji R, Wells P, et al. Rivaroxaban for treatment of suspected or confirmed heparin-induced thrombocytopenia study. Journal of Thrombosis and Haemostasis. 2016;14(6):1206-10. 
10. Sharifi M, Bay C, Vajo Z, Freeman W, Sharifi M, Schwartz F. New Oral Anticoagulants in the Treatment of Heparin- Induced Thrombocytopenia. Thrombosis Research. 2015;135(4):607-9. 